Perspective

\title{
Subjective memory complaints as a predictor of mild cognitive impairment and Alzheimer's disease
}

\author{
Samuel L. Warren ${ }^{1}$ (D) Edwina Reid ${ }^{2} \cdot$ Paige Whitfield $^{2} \cdot$ Ahmed A. Moustafa $^{1,3}$ \\ Received: 5 January 2022 / Accepted: 12 February 2022 \\ Published online: 17 February 2022 \\ (c) The Author(s) 2022 OPEN
}

\begin{abstract}
While there is a multitude of studies on mild cognitive impairment ( $\mathrm{MCl}$; more than 80,000 articles), subjective memory complaints (SMC) have received less attention as a prodromal stage of Alzheimer's disease (AD; less than 2000 articles). In this perspective review article, we argue that SMC should also be considered as another risk factor for the development of $A D$, and perhaps a pre- $\mathrm{MCl}$ condition. This recognition of $S M C$ could help clinicians to identify individuals at risk of developing dementia and could provide protective treatment for them. Accordingly, in this perspective article, we review key studies that outline the nature of SMC, discuss how SMC is measured, explore SMC in $\mathrm{MCl}$, introduce some approaches to SMC treatment, and we discuss future directions for SMC research. Overall, we argue that, like $\mathrm{MCl}$, there should be more research on SMC as a risk factor for developing AD. Consequentially, we aim to highlight the need for further research on SMC and the condition's role as a potential neuroprotector against AD (e.g., early-stage marker).
\end{abstract}

Keywords Alzheimer's disease (AD) · Mild cognitive impairment (MCI) · Subjective memory complaints (SMC) - Episodic memory

\section{Introduction}

Alzheimer's disease (AD) is a neurodegenerative disorder with an insidious onset that progresses through cognitive, functional, and behavioural impairment [1, 2]. AD is the second leading cause of death in Australia, accounting for $70-80 \%$ of dementia diagnoses [3]. Generally, by the time AD is diagnosed, the neurological damage caused by amyloid plaques and neurofibrillary tangles is deemed irreversible [4]. Despite the overwhelming burden of disease, the cause of AD is unknown, and treatments are largely unsuccessful [5]. Therefore, as the population continues to age, society is increasingly faced with an impending public health crisis [6]. Consequently, there is a critical need for AD detection methods that can identify at-risk individuals early in the disease progression and help facilitate effective treatment.

In the literature, researchers often focus on pre-dementia conditions, such as mild cognitive impairment (MCl), to observe $A D$ progression and identify at-risk individuals. $\mathrm{MCl}$ is the transitional stage between normative aging and dementia, where an individual can maintain most functional abilities despite evidence of neuropsychological impairment $[7,8]$. In turn, $\mathrm{MCl}$ is recognised as an important risk state for $\mathrm{AD}$ [7]. The prevalence of $\mathrm{MCl}$ in adults over the age of 65 is reported to be anywhere between 3 and $36 \%[7,9]$. Individuals diagnosed with $\mathrm{MCl}$ are at higher risk of progression to dementia, with an estimated $11-33 \%$ of individuals progressing to AD dementia within two years $[6,7]$. Accordingly, $\mathrm{MCl}$

$\triangle$ Samuel L. Warren, SamuelLWarren@outlook.com | 1 School of Psychology, Faculty of Society and Design, Bond University, Gold Coast, QLD, Australia. ${ }^{2}$ School of Psychology, Western Sydney University, Sydney, NSW, Australia. ${ }^{3}$ Department of Human Anatomy and Physiology, The Faculty of Health Sciences, University of Johannesburg, Johannesburg, South Africa.

Discover Psychology $\quad$ (2022) 2:13 $\quad$ https://doi.org/10.1007/s44202-022-00031-9 
enables researchers to easily study a large sample of individuals that will progress to AD. In turn, there is a vast literature that examines $\mathrm{MCl}$ as a predictor or transitional stage of $\mathrm{AD}$. For Example, psychological tests have been devised that selectively focus on the cognitive domains affected by $\mathrm{MCl}$ and dementia to separate healthy individuals from those with clinical symptoms $[9,10]$. Nonetheless, evidence suggests that $\mathrm{MCl}$ is not the earliest stage of prodromal $\mathrm{AD}$ and that earlier disease detection is necessary for significant intervention [11, 12].

In 2018, the National Institute on Aging and Alzheimer's Association (NIA-AA) included subjective experiences of cognitive decline as a transitional phase between healthy cognition and preclinical dementia $[4,13-15]$. Increasingly, evidence suggests that subjective memory complaints (SMC) may represent the first preclinical manifestation of AD or capture a 'pre-MCl' stage [11, 12]. Accordingly, SMC is a critical area of research that could help researchers to better understand, predict, and treat AD. However, SMC lacks substantive research in comparison to other similar conditions such as $\mathrm{MCI}$. For example, a search for MCI returns approximately 80,000 results in the PubMed database while the term SMC nets less than 2000 results. Accordingly, in this perspective article, we review key papers associated with SMC and the prediction of $\mathrm{MCl}$ or AD. These articles were chosen via our research team for review in summer 2021 while situated at Western Sydney University. Each article was found using online databases (e.g., PubMed) and was included via reviewer consensus if the paper discussed SMC as a predictor or prodromal stage of $\mathrm{MCl}$ or AD. Accordingly, this perspective review aims to discuss SMC as a precursor to dementia and argue that it is a critical area of AD research. In turn, we outline the nature of SMC, discuss how SMC is measured, explore the presence of SMC in individuals with $\mathrm{MCl}$, introduce some approaches to SMC treatment, and discuss future directions for SMC research. Consequently, we aim to highlight the current literature on $S M C$, discuss how $S M C$ is a risk factor or transitional stage for $M C l$ and $A D$, and stress avenues for future research.

\section{What is SMC?}

Many individuals report noticing subtle changes in memory and cognitive function before the detection of cognitive impairment (e.g., MCl) [16]. SMC has therefore been highlighted as important for diagnosing prodromal AD [17, 18]. SMC is defined as the subjective perception of cognitive decline compared to previous levels of functioning in individuals with normal cognition $[17,19]$. Individuals with SMC experience subjective problems or changes with their memory that are rarely measurable by traditional cognitive tests [20]. These complaints can be present in individuals with cognitive impairment, such as those with amnestic or non-amnestic $\mathrm{MCl}$, as well as in people with no objective cognitive decline. However, individuals with these complaints usually have greater objective memory decline, as well as lower overall cognitive performance and perceived health than individuals without SMC [21]. The relationship between SMC and actual memory function is not always clear. This lack of understanding may occur because SMC represents subtle cognitive changes that are small enough that they cannot be detected by standard cognitive assessments [22]. SMC is described elsewhere in the literature as subjective cognitive decline (SCD), subjective memory impairment (SMI), and subjective cognitive complaint (SCC). All these terms are different definitions of the same concept and generally refer to an individual's subjective rating of their cognitive ability [23]. In this paper, we use the term SMC to encompass the overarching subjective experience of cognitive decline.

SMC is relatively common in older adults and increases in numbers and severity as people age. A review by Brigola et al. [24] found that approximately $50 \%$ of people aged 50 to 59 experience SMC, as do $63 \%$ of 80 to 100 -year-olds. Brigola et al. [24] also found that there is an association between SMC and cognitive impairment. Specifically, they found that in some individuals, SMC was predictive of dementia at a two year follow up. In another study, Palmer et al. [25] found that $51 \%$ of people who developed dementia had SMC at baseline. Accordingly, it is suggested that SMC may be predictive of a progression to dementia. For example, Parfenov et al. [19] found that individuals who reported SMC were 2.15 times more likely to develop $\mathrm{MCl}$ and 2.17 times more likely to develop dementia than healthy controls. Furthermore, when compared to healthy controls, individuals with SMC have a greater risk of AD-associated biomarker abnormalities, regional brain hypometabolism, and atrophy of the medial temporal lobe [19, 26-28].

In a meta-analysis, Mitchell [14] showed that SMC is found in $42.8 \%$ of individuals with dementia and $38.2 \%$ of those with $\mathrm{MCl}$, compared to a rate of $17.4 \%$ in healthy controls. The rate of SMC was significantly higher in dementia than in $\mathrm{MCl}$. This meta-analysis also determined that the presence or lack of SMC alone could be an effective way to rule out a diagnosis of dementia or $\mathrm{MCl}$. This discernment of dementia could be an important clinical application of SMC. However, it is difficult to draw such a causal link because SMC-related decline can also be related to other factors such as an individual's emotional functioning and personality [29]. SMC research is further complicated by an estimated $60 \%$ of people who have cognitive impairments but do not report SMC [18]. It is unclear whether these missed reports are a 
mistake (e.g., lack of awareness of SMC) or because these individuals do not perceive SMC-related changes as significant or requiring attention.

Current epidemiological studies have found SMC prevalence to be $88 \%$ in individuals aged over 85 years, 25 to $50 \%$ in individuals over 65 years, and $20 \%$ in individuals under 65 years of age [23]. Evidence also suggests that SMC is linked to increased risk of $\mathrm{MCl}$ and $\mathrm{AD}[30,31]$. Accordingly, SMC must be further studied as a transitional stage or risk factor for AD. In turn, advancements in SMC research could lead to the development of detection methods that can identify individuals with SMC and direct future treatment [32]. Research into SMC is also important to other domains beyond $A D$ treatment and progression. For example, research into SMC could also contribute to other topics of interest such as memory decline, $A D$ biomarkers, and disease prediction. Further research may also be beneficial not only to those with memory or cognitive impairments but also to individuals who experience SMC without associated cognitive decline or impairment. For example, Miley-Akerstedt et al. [32] found that individuals with SMC and no objective cognitive decline had more years of education, more sleep problems, and a lot to do in their daily lives when compared to those who had SMC and objective memory problems. These participants also had increased beta-amyloid levels in their cerebrospinal fluid, indicating they had a biomarker profile that was nonpathological. Accordingly, SMC must be further researched to understand the condition and its links to $A D$.

\section{Measuring SMC}

Measures of SMC are generally neuropsychological tests in which individuals rate their current memory, compare current and previous memory performance, or rate their memory in comparison to peers $[18,30]$. Tests of SMC have been found to predict accelerated cognitive decline; however, research into the relationship between SMC and objective performance on cognitive measures is limited [10,27]. Nonetheless, longitudinal testing has shown that individuals with $\mathrm{SMC}$ are more likely to progress to $\mathrm{MCl}$ and dementia than those without SMC [33, 34].

While it is well researched that $M C I$ precedes $A D$, the significance of SMC in the continuum remains unclear [19]. This is due, in part, to the fact that SMC is not clearly defined or uniformly measured in the literature [30]. Assessments of SMC generally include brief questions regarding an individual's perceived memory function as well as how changes in memory may be affecting daily living [18]. However, these measures are not always comparative and may ask individuals to perform various tasks such as rating their current memory, comparing current and previous memory performance, or rating their memory in comparison to peers [30]. This variability makes comparing study results difficult and can lead to the interference of other variables such as anxiety and depression [30, 35, 36].

Rabin et al. [36] conducted a systematic review of self-report measures of SMC. Their results found that 34 different self-report measures were used in SMC research and that studies varied significantly in the method, range, format, and timeframe when assessing the condition [36]. They also found that the Memory Complaint Questionnaire (MAC-Q) [37] and the Measurement of Everyday Cognition (ECog) [38] were the most common tests used to assess SMC. In another study, Opdebeeck et al. [35] compared the agreement of five different SMC measures in identifying individuals over the age of 65 with SMC. Opdebeeck et al. [35] found that there was limited overlap in the ability of tests to identify individuals with SMC. Critically, they found that only one individual (out of 204 participants) with SMC was identified by all five measures. This finding highlighted that no scale fully encapsulates SMC and that different measures address different symptoms of the condition. These results are worrying as SMC measures are used interchangeably in clinical practice and research. Accordingly, there is conflicting evidence regarding the ability of neuropsychological tests to measure SMC. We suggest that this contention may occur due to a lack of consistent definition and operationalisation of SMC.

While there is some argument about the validity of some SMC measures, there are also some tests that are known to accurately detect SMC. For example, some scales_-such as the Subjective Memory Complaints Questionnaire (SMCQ)—have been specifically developed to evaluate the level of cognitive impairment in people presenting with memory complaints [39]. However, these measures are not widely used because most SMC research is conducted in reference to AD. In turn, valid tests such as the SMCQ are disregarded for popular tests of AD or MCI (e.g., MMSE). Accordingly, it is important to use valid tests for SMC when possible; however, it is also important to determine if more commonly used tests can detect behavioural, clinical, and cognitive differences between those with SMC and individuals in the later stages of cognitive decline. By understanding the capacity of current AD tests, researchers would be able to know whether they can assess $S M C, M C l$, and $A D$ with one test or whether separate scales for $S M C$ are required. These findings would drastically improve the validity of $S M C$ and $A D$ research. 


\section{$4 \mathrm{SMC}$ in $\mathrm{MCl}$}

Individuals with SMC experience subjective problems or changes in their memory. These complaints can be present in individuals with cognitive impairment, such as those with amnestic or non-amnestic $\mathrm{MCl}$, as well as in people without $\mathrm{MCl}$. One of the greatest differences between $\mathrm{MCl}$ and $\mathrm{SMC}$ is that, while memory complaints can be present in individuals with both conditions, objective memory decline can only be conclusively determined in those with $\mathrm{MCI}$ [14]. Accordingly, SMC is proposed to precede $\mathrm{MCl}$ and both conditions are recognised as prodromal phases of AD [40]. In the literature, $S M C$ is regularly used as a measure when detecting $\mathrm{MCl}$ in research studies [18]. However, the inclusion of $\mathrm{SMC}$ in $\mathrm{MCl}$ definitions is deemed controversial, with research highlighting that SMC measures may lack sensitivity and specificity as a criterion for $\mathrm{MCl}$ [41]. Accordingly, definitions of $\mathrm{MCl}$ also require a measure of objective impairment in cognition, intact general cognitive functioning, and absence of dementia [18]. The inclusion of SMC in the classification of $\mathrm{MCl}$ reduces the prevalence of $\mathrm{MCl}$ as research has found that up to $62 \%$ of patients experiencing cognitive decline do not report SMC [18]. Explanations for this discrepancy include an individual's ability to adapt to and cope with cognitive decline, where some people do not perceive change as significant [18].

One key area of research involving SMC and $\mathrm{MCl}$ is the effects of neuropsychiatric symptoms on the progression of these conditions. For example, Yates et al. [18] conducted a study to clarify the relationship between mood and SMC in people with $\mathrm{MCl}$. They also sought to determine whether SMC is a prominent feature of $\mathrm{MCl}$ and whether SMC should be included in the definition of $\mathrm{MCl}$. Yates et al. [18] assessed both mood and SMC in participants from the First Cognitive Function and Ageing Study funded by the Medical Research Council (MRC-CFAS). SMC was self-reported by the participant and included memory problems like difficulties with memory or increased forgetfulness. At baseline, 557 out of 1344 participants reported SMC. Moreover, all participants with $\mathrm{MCl}$ reported SMC, and there were no SMC present for individuals who had $\mathrm{MCl}$ without SMC due to study definitions. Yates et al. [18] showed that there was a significant relationship between $\mathrm{SMC}, \mathrm{MCl}$, and anxiety or depression at baseline. Specifically, they found that the presence of anxiety or depression (at baseline and at follow up) had a significant relationship, depression was associated with a change in cognitive status, and anxiety was not related to a change in cognitive status.

However, there is some contention concerning the relationship between SMC and neuropsychiatric disorders when assessing for $\mathrm{MCl}$. In the same study, Yates et al. [18] found that anxiety and depression were more strongly related to SMC than objective cognitive impairment. Accordingly, Yates et al. [18] concluded that SMC may be a symptom of anxiety and depression, rather than related to objective cognitive function (e.g., $\mathrm{MCl}$ ). As a result, Yates et al. [18] questioned whether $\mathrm{SMC}$ should be a criterion in the definitions of $\mathrm{MCl}$. Consequently, this research highlights the need for the standardisation of SMC measures and definitions. Researchers should also seek to longitudinally research neuropsychiatric symptoms and their association with preclinical $A D, S M C, M C l$, and $A D$ dementia.

\section{SMC treatment}

The identification of SMC could play a crucial role in the treatment and diagnosis of AD [32]. For example, individuals with SMC may not require a full AD or dementia evaluation and could be referred to treatment. We hypothesise that treatment for SMC would likely include psychological interventions targeted at reducing stress, improving sleep, and trying to reduce their workload. If modifying these factors does not resolve SMC, an AD or dementia assessment could then be warranted. In turn, these SMC screenings could reduce the need to refer all patients with memory concerns to specialist dementia clinics, could reduce the costs of unnecessary procedures, and may lead to an improvement in their symptoms regardless of the presence of cognitive impairment.

Given the potential of these SMC diagnoses to improve AD screening, it is also important to investigate interventions for individuals with SMC. There is lacking research on interventions for SMC, yet some studies do exist. For example, Metternich et al. [42] investigated the effect of memory training (MT) and expectancy change interventions (EC) on SMC. MT and EC are aimed at changing an individual's beliefs and attitudes about their memory through psychoeducation, cognitive restructuring, or physical training. Metternich et al. [42] demonstrated that EC interventions had significant effects on SMC compared to those on a waitlist or in a placebo group. However, they did find discrepancies between the treatment conditions. Specifically, Metternich et al. [42] concluded that EC interventions are likely to be more effective than MT in improving subjective memory. However, MT was the only intervention type that positively affected objective memory performance. Accordingly, Metternich et al. [42] suggested that MT does have some benefit as an intervention 
for those with memory difficulties. MT was also shown to be the preferred intervention by individuals with SMC compared to psychological interventions like EC, or medication. These results may demonstrate that a combination of MT and EC is likely to be more effective for SMC treatment. This study also establishes the need for further research into SMC and its treatment, as it is important to understand whether treating neuropsychiatric symptoms in those with SMC, treating SMC alone, or both, has benefits for those with SMC, and whether treatment impacts on progression to $\mathrm{MCl}$ or $\mathrm{AD}$.

\section{Conclusions and future directions}

In this paper, we have outlined the concept of SMC, discussed current measures for SMC, discussed SMC in $\mathrm{MCl}$, and touched on some approaches to SMC treatment. Throughout all these topics there has been a constant theme of promise and contention. Specifically, prior research indicates that SMC is a promising marker of early-stage AD that could be used to progress dementia research; However, corresponding research also states that SMC is poorly understood, measured, and investigated. In this study, we present both the contention and promise of SMC to encourage further investigation. As a conclusion to this paper, we will discuss areas for further research.

To progress SMC research, four main problems must be overcome. Firstly, there is a lacking standardisation of SMC. As previously discussed, SMC has multiple names and definitions throughout the literature. These different definitions can affect the generalisability and the operationalisation of SMC research. In turn, SMC-like concepts must be properly defined and consistent throughout the literature. Such standardisation could result in more valid, generalisable, and reliable research. Secondly, there is a lack of longitudinal SMC research. Longitudinal research is required to understand the progression of the condition, its comorbidities, and its role in AD progression. Without this long-term investigation, there will continue to be confusion about the role of SMC in cognitive and neurodegenerative decline. Studies such as Opdebeeck et al. [35] have also suggested that longitudinal studies could help standardise SMC definitions.

Thirdly, clinical tests for SMC are often misused and misunderstood. Further research is required to understand the ability of tests to measure SMC and AD-related disorders (as discussed in Sect. 3). Significant research is also required to identify which tests should be used to diagnose and monitor SMC. In turn, innovations in SMC testing could result in a better understanding of the condition as well as stronger screening and treatment strategies. Finally, the last common limitation of SMC research is that it lacks an understanding of the condition's comorbidities and relationships. For example, it is understood that $S M C$ is a precursor to $A D$, however, the relationship between $S M C, M C I$, and neuropsychiatric disorders is not understood. Accordingly, there is a critical need for research that investigates the interactions between SMC and markers of AD. Moreover, there is also a need to understand the role of SMC in the AD continuum and its relationships to the stages of AD.

In conclusion, the standardisation, longitudinal analysis, development of testing material, and investigation into SMC's relationships are all key areas of research. We hope that future investigation can properly detect, treat, and understand SMC as part of the AD continuum. We also hope that further research can use SMC to detect early-stage AD and help improve the lives of individuals with dementia.

Authors' contributions PW and ER wrote sections of this paper as part of their clinical master's thesis. AAM was the primary supervisor on the thesis, and SLW was involved in the thesis development. Following thesis submission, AAM wrote the first draft of the paper, and SLW worked on all follow-up drafts, editing, and the papers submission. All authors read and approved the final manuscript.

Funding This study would like to acknowledge funding received as part of the Australian Governments Research Training Program Scholarship.

Data availability Not applicable.

Code availability Not applicable.

Declarations

Ethics approval and consent to participate Not applicable.

Consent for publication Not applicable.

Competing interests The authors declare no competing interests. 
Open Access This article is licensed under a Creative Commons Attribution 4.0 International License, which permits use, sharing, adaptation, distribution and reproduction in any medium or format, as long as you give appropriate credit to the original author(s) and the source, provide a link to the Creative Commons licence, and indicate if changes were made. The images or other third party material in this article are included in the article's Creative Commons licence, unless indicated otherwise in a credit line to the material. If material is not included in the article's Creative Commons licence and your intended use is not permitted by statutory regulation or exceeds the permitted use, you will need to obtain permission directly from the copyright holder. To view a copy of this licence, visit http://creativecommons.org/licenses/by/4.0/.

\section{References}

1. Marshall GA, Amariglio RE, Sperling RA, Rentz DM. Activities of daily living: where do they fit in the diagnosis of Alzheimer's disease? Neurodegener Dis Manag. 2012;2:483-91. https://doi.org/10.2217/nmt.12.55.

2. Mendez MF. The relationship between anxiety and Alzheimer's disease. J Alzheimers Dis Rep. 2021;5:171-7. https://doi.org/10.3233/ ADR-210294.

3. Australian Bureau of Statistics, Causes of death, Australia, Australian Bureau of Statistics Canberra, 2019.

4. Jack CR, Bennett DA, Blennow K, Carrillo MC, Dunn B, Haeberlein SB, Holtzman DM, Jagust W, Jessen F, Karlawish J, Liu E, Molinuevo JL, Montine T, Phelps C, Rankin KP, Rowe CC, Scheltens P, Siemers E, Snyder HM, Sperling R, Elliott C, Masliah E, Ryan L, Silverberg N. NIA-AA research framework: toward a biological definition of Alzheimer's disease. Alzheimers Dement. 2018;14:535-62. https://doi.org/10.1016/j. jalz.2018.02.018.

5. Alvin Ang TF, Joshi P, Au R. Vascular risk factors and their relationship to brain aging: findings from the Framingham Heart Study. In: Vascular disease, Alzheimer's disease, and mild cognitive impairment, Oxford University Press, New York, 2020. https://doi.org/10.1093/ oso/9780190634230.003.0002.

6. Eppig J, Werhane M, Edmonds EC, Wood L-D, Bangen KJ, Jak A, Thomas KR, Wong C, Weigand A, Bondi MW. Neuropsychological contributions to the diagnosis of mild cognitive impairment associated with Alzheimer's disease. In: Vascular Disease, Alzheimer's Disease, and Mild Cognitive Impairment, Oxford University Press, New York, 2020. https://doi.org/10.1093/oso/9780190634230.003.0004.

7. Ma L. Depression, anxiety, and apathy in mild cognitive impairment: current perspectives. Front Aging Neurosci. 2020;12:9. https://doi. org/10.3389/fnagi.2020.00009.

8. Petersen RC, Morris JC. Mild cognitive impairment as a clinical entity and treatment target. Arch Neurol. 2005;62:1160-3. https://doi.org/ 10.1001/archneur.62.7.1160.

9. Breton A, Casey D, Arnaoutoglou NA. Cognitive tests for the detection of mild cognitive impairment $(\mathrm{MCl})$, the prodromal stage of dementia: meta-analysis of diagnostic accuracy studies. Int J Geriatr Psychiatry. 2019;34:233-42. https://doi.org/10.1002/gps.5016.

10. Warren SL, Moustafa AA, Alashwal H. Harnessing forgetfulness: can episodic-memory tests predict early Alzheimer's disease? Exp Brain Res. 2021. https://doi.org/10.1007/s00221-021-06182-w.

11. Choe YM, Byun MS, Lee JH, Sohn BK, Lee DY, Kim JW. Subjective memory complaint as a useful tool for the early detection of Alzheimer's disease. Neuropsychiatr Dis Treat. 2018;14:2451-60. https://doi.org/10.2147/NDT.S174517.

12. Jessen $F$, Amariglio RE, van Boxtel $M$, Breteler $M$, Ceccaldi $M$, Chételat $G$, Dubois $B$, Dufouil $C$, Ellis KA, van der Flier WM, Glodzik $L$, van Harten AC, de Leon MJ, McHugh P, Mielke MM, Molinuevo JL, Mosconi L, Osorio RS, Perrotin A, Petersen RC, Rabin LA, Rami L, Reisberg B, Rentz DM, Sachdev PS, de la Sayette V, Saykin AJ, Scheltens P, Shulman MB, Slavin MJ, Sperling RA, Stewart R, Uspenskaya O, Vellas B, Visser PJ, Wagner M, Subjective Cognitive Decline Initiative (SCD-I) Working Group. A conceptual framework for research on subjective cognitive decline in preclinical Alzheimer's disease. Alzheimers Dement. 2014;10:844-52. https://doi.org/10.1016/j.jalz.2014.01.001.

13. Abdulrab K, Heun R. Subjective memory impairment. A review of its definitions indicates the need for a comprehensive set of standardised and validated criteria. Eur Psychiatry. 2008;23:321-30. https://doi.org/10.1016/j.eurpsy.2008.02.004.

14. Mitchell AJ. The clinical significance of subjective memory complaints in the diagnosis of mild cognitive impairment and dementia: a meta-analysis. Int J Geriatr Psychiatry. 2008;23:1191-202. https://doi.org/10.1002/gps.2053.

15. Mitchell AJ. Is it time to separate subjective cognitive complaints from the diagnosis of mild cognitive impairment? Age Ageing 2008;37:497-9. https://doi.org/10.1093/ageing/afn147.

16. Aisen PS, Cummings J, Jack CR, Morris JC, Sperling R, Frölich L, Jones RW, Dowsett SA, Matthews BR, Raskin J, Scheltens P, Dubois B. On the path to 2025: understanding the Alzheimer's disease continuum. Alzheimers Res Ther. 2017;9:60. https://doi.org/10.1186/ s13195-017-0283-5.

17. Liew TM. Subjective cognitive decline, anxiety symptoms, and the risk of mild cognitive impairment and dementia. Alzheimers Res Therapy. 2020;12:107. https://doi.org/10.1186/s13195-020-00673-8.

18. Yates JA, Clare L, Woods RT. Subjective memory complaints, mood and MCl: a follow-up study. Aging Ment Health. 2017;21:313-21. https:// doi.org/10.1080/13607863.2015.1081150.

19. Parfenov VA, Zakharov VV, Kabaeva AR, Vakhnina NV. Subjective cognitive decline as a predictor of future cognitive decline: a systematic review. Dement Neuropsychol. 2020;14:248-57. https://doi.org/10.1590/1980-57642020dn14-030007.

20. Hsieh S-W, Hsiao S-F, Liaw L-J, Huang L-C, Yang Y-H. Effects of multiple training modalities in the elderly with subjective memory complaints. Medicine (Baltimore). 2019;98: e16506. https://doi.org/10.1097/MD.0000000000016506.

21. Carrasco PM, Montenegro-Peña M, López-Higes R, Estrada E, Prada Crespo D, Montejo Rubio C, García Azorín D. Subjective memory complaints in healthy older adults: fewer complaints associated with depression and perceived health, more complaints also associated with lower memory performance. Arch Gerontol Geriatr. 2017;70:28-37. https://doi.org/10.1016/j.archger.2016.12.007.

22. Kawagoe T, Onoda K, Yamaguchi S. Subjective memory complaints are associated with altered resting-state functional connectivity but not structural atrophy. Neurolmage Clin. 2019;21: 101675. https://doi.org/10.1016/j.nicl.2019.101675.

23. Si T, Xing G, Han Y. Subjective cognitive decline and related cognitive deficits. Front Neurol. 2020;11:247. https://doi.org/10.3389/fneur. 2020.00247. 
24. Brigola AG, Manzini CSS, Oliveira GBS, Ottaviani AC, Sako MP, Vale FAC. Subjective memory complaints associated with depression and cognitive impairment in the elderly: a systematic review. Dement Neuropsychol. 2015;9:51-7. https://doi.org/10.1590/S1980-57642 015 DN91000009.

25. Palmer K, Bäckman L, Winblad B, Fratiglioni L. Detection of Alzheimer's disease and dementia in the preclinical phase: population based cohort study. BMJ. 2003;326:245. https://doi.org/10.1136/bmj.326.7383.245.

26. Mosconi L, De Santi S, Brys M, Tsui WH, Pirraglia E, Glodzik-Sobanska L, Rich KE, Switalski R, Mehta PD, Pratico D, Zinkowski R, Blennow $\mathrm{K}$, de Leon MJ. Hypometabolism and altered cerebrospinal fluid markers in normal apolipoprotein E E4 carriers with subjective memory complaints. Biol Psychiatry. 2008;63:609-18. https://doi.org/10.1016/j.biopsych.2007.05.030.

27. Samieri C, Proust-Lima C, Glymour MM, Okereke OI, Amariglio RE, Sperling RA, Rentz DM, Grodstein F. Subjective cognitive concerns, episodic memory, and the APOE $\varepsilon 4$ allele. Alzheimers Dement. 2014;10:752-759.e1. https://doi.org/10.1016/j.jalz.2014.06.012.

28. Saykin AJ, Wishart HA, Rabin LA, Santulli RB, Flashman LA, West JD, McHugh TL, Mamourian AC. Older adults with cognitive complaints show brain atrophy similar to that of amnestic MCl. Neurology. 2006;67:834-42. https://doi.org/10.1212/01.wnl.0000234032.77541.a2.

29. Norman AL, Woodard JL, Calamari JE, Gross EZ, Pontarelli N, Socha J, DeJong B, Armstrong K. The fear of Alzheimer's disease: mediating effects of anxiety on subjective memory complaints. Aging Ment Health. 2018;24:308-14. https://doi.org/10.1080/13607863.2018.15340 81.

30. Hill NL, Mogle J, Bhargava S, Bell TR, Bhang I, Katz M, Sliwinski MJ. Longitudinal relationships among depressive symptoms and three types of memory self-report in cognitively intact older adults. Int Psychogeriatr. 2020;32:719-32. https://doi.org/10.1017/S104161021 $900084 X$.

31. Seo EH, Kim H, Choi KY, Lee KH, Choo IH. Association of subjective memory complaint and depressive symptoms with objective cognitive functions in prodromal Alzheimer's disease including pre-mild cognitive impairment. J Affect Disord. 2017;217:24-8. https://doi.org/10. 1016/j.jad.2017.03.062.

32. Miley-Akerstedt A, Jelic V, Marklund K, Walles H, Åkerstedt T, Hagman G, Andersson C. Lifestyle factors are important contributors to subjective memory complaints among patients without objective memory impairment or positive neurochemical biomarkers for Alzheimer's disease. Dement Geriatr Cogn Disord Extra. 2018;8:439-52. https://doi.org/10.1159/000493749.

33. Jessen F, Wiese B, Bachmann C, Eifflaender-Gorfer S, Haller F, Kölsch H, Luck T, Mösch E, van den Bussche H, Wagner M, Wollny A, Zimmermann T, Pentzek M, Riedel-Heller SG, Romberg H-P, Weyerer S, Kaduszkiewicz H, Maier W, Bickel H, German Study on Aging, Cognition and Dementia in Primary Care Patients Study Group. German study on aging, prediction of dementia by subjective memory impairment: effects of severity and temporal association with cognitive impairment. Arch Gen Psychiatry. 2010;67:414-22. https://doi.org/10.1001/ archgenpsychiatry.2010.30.

34. Stewart R, Godin O, Crivello F, Maillard P, Mazoyer B, Tzourio C, Dufouil C. Longitudinal neuroimaging correlates of subjective memory impairment: 4-year prospective community study. Br J Psychiatry. 2011;198:199-205. https://doi.org/10.1192/bjp.bp.110.078683.

35. Opdebeeck C, Yates JA, Kudlicka A, Martyr A. What are subjective cognitive difficulties and do they matter? Age Ageing. 2019;48:122-7. https://doi.org/10.1093/ageing/afy148.

36. Rabin LA, Smart CM, Crane PK, Amariglio RE, Berman LM, Boada M, Buckley RF, Chételat G, Dubois B, Ellis KA, Gifford KA, Jefferson AL, Jessen F, Katz MJ, Lipton RB, Luck T, Maruff P, Mielke MM, Molinuevo JL, Naeem F, Perrotin A, Petersen RC, Rami L, Reisberg B, Rentz DM, Riedel-Heller SG, Risacher SL, Rodriguez O, Sachdev PS, Saykin AJ, Slavin MJ, Snitz BE, Sperling RA, Tandetnik C, van der Flier WM, Wagner M, Wolfsgruber S, Sikkes SAM, The A.D.N. Initiative. Subjective cognitive decline in older adults: an overview of self-report measures used across 19 international research studies. J Alzheimers Dis. 2015;48:S63-86. https://doi.org/10.3233/JAD-150154.

37. Crook TH, Feher EP, Larrabee GJ. Assessment of memory complaint in age-associated memory impairment: the MAC-Q. Int Psychogeriatr. 1992;4:165-76. https://doi.org/10.1017/s1041610292000991.

38. Farias ST, Mungas D, Reed BR, Cahn-Weiner D, Jagust W, Baynes K, Decarli C. The measurement of everyday cognition (ECog): scale development and psychometric properties. Neuropsychology. 2008;22:531-44. https://doi.org/10.1037/0894-4105.22.4.531.

39. Duman B, Özel Kızıl ET, Baran Z, Kırıcı S, Turan E. Discrepancy between subjective memory complaints and objective memory deficits in elderly patients with depression compared to mild cognitive impairment. Turk Psikiyatri Derg Turk J Psychiatry. 2016;27:1-7.

40. Eliassen CF, Reinvang I, Selnes P, Grambaite R, Fladby T, Hessen E. Biomarkers in subtypes of mild cognitive impairment and subjective cognitive decline. Brain Behav. 2017;7: e00776. https://doi.org/10.1002/brb3.776.

41. Lenehan ME, Klekociuk SZ, Summers MJ. Absence of a relationship between subjective memory complaint and objective memory impairment in mild cognitive impairment $(\mathrm{MCl})$ : is it time to abandon subjective memory complaint as an $\mathrm{MCl}$ diagnostic criterion? Int Psychogeriatr. 2012;24:1505-14. https://doi.org/10.1017/S1041610212000695.

42. Metternich B, Kosch D, Kriston L, Härter M, Hüll M. The effects of nonpharmacological interventions on subjective memory complaints: a systematic review and meta-analysis. Psychother Psychosom. 2010;79:6-19. https://doi.org/10.1159/000254901.

Publisher's Note Springer Nature remains neutral with regard to jurisdictional claims in published maps and institutional affiliations. 